\title{
Crescimento, produção de fitomassa e teor de óleo essencial de melissa (Melissa officinalis L.) sob diferentes níveis de sombreamento
}

\author{
MEIRA, M.R.*; MARTINS, E.R.; MANGANOTTI, S.A. \\ Instituto de Ciências Agrárias da Universidade Federal de Minas Gerais, Av. Universitária, 1000, CEP: 39400-000, \\ Montes Claros-Brasil *messulan@hotmail.com
}

\begin{abstract}
RESUMO: O objetivo foi avaliar a variação no teor de óleo essencial, crescimento e produção de fitomassa de Melissa officinalis L. cultivada sob diferentes níveis de sombreamento. O delineamento experimental foi inteiramente ao acaso (DIC) com quatro níveis de radiação fotossinteticamente ativa (RFA), luz plena (testemunha) e sombreamento progressivo com tela tipo sombrite (sombreamento de 75,50 e $25 \%$ ) e seis repetições. Foram avaliados: diâmetro do caule, altura da planta, massa fresca e seca da parte aérea, teor do óleo essencial, rendimento de óleo e área foliar. Os dados foram submetidos à análise de variância e análise de regressão a 1 e $5 \%$ de probabilidade de erro. As características, diâmetro do caule e altura da planta, não apresentaram diferenças significativas entre os tratamentos. A produção de massa fresca, massa seca e o teor de óleo essencial, foram similares entre todos os tratamentos diferenciando apenas do tratamento em pleno sol, onde (MF): $25 \%=50 \%, 91,33 \mathrm{~g}$ e $68,66 \mathrm{~g}$; (MS): $25 \%=0 \%=50 \%, 11,35,11,66$, 10,66 g e 4,33 g e TO: $25 \%=50 \%, 0,48$ e 0,40\% respectivamente. O tratamento que resultou em menor produtividade foi o sombreado a $75 \%$. Assim, nas condições norte-mineiras, de fevereiro a junho, o cultivo da melissa pode ser conduzido em condições de até $50 \%$ de sombreamento, favorecendo a produção de massa seca, massa fresca e teor de óleo essencial; sendo estas variáveis de interesse comercial pelos produtores de plantas medicinais e aromáticas.
\end{abstract}

Palavras-chave: planta medicinal, metabólito secundário, produção, intensidade luminosa

\begin{abstract}
Growth, biomass production and essential oil content of lemon balm (Melissa officinalis L.) under different shading levels. The aim of this study was to evaluate the variation in essential oil content, growth and biomass production of Melissa officinalis L. cultivated under different shading levels. Experimental design was completely randomized (CRD) with four levels of photosynthetically active radiation (PAR), full light (control) and progressive shading with shade net (shading of 75,50 and $25 \%$ ) and six replicates. The following parameters were assessed: stem diameter, plant height, fresh mass and dry mass of shoot, essential oil content, essential oil yield and leaf area. Data were subjected to analysis of variance and regression analysis at 1 and $5 \%$ error probability. The characteristics stem diameter and plant height did not show significant differences among treatments. Production of fresh weight, dry mass and essential oil content was similar among all treatments, except for full light, where (FW): $25 \%=50 \%, 91.33 \mathrm{~g}$ and $68.66 \mathrm{~g}$; (DW): $25 \%=0 \%=50 \%, 11.35,11.66,10.66 \mathrm{~g}$ and $4.33 \mathrm{~g}$ and OC: $25 \%=50 \%, 0.48$ and $0.40 \%$ respectively. The treatment that resulted in the lowest productivity was $75 \%$ shading. Thus, in the north of Minas Gerais State, Brazil, from February to June, lemon balm can be cultivated under conditions of up to $50 \%$ shading, favoring the production of dry mass, fresh mass and essential oil content; these variables are of commercial interest for producers of medicinal and aromatic plants.
\end{abstract}

Key words: medicinal plant, secondary metabolite, production, light intensity

\section{INTRODUÇÃO}

A melissa (Melissa officinalis L.), conhecida como erva-cidreira verdadeira pertence à família Lamiaceae, é de origem asiática e europeia e foi introduzida no Brasil há mais de um século, sendo atualmente cultivada em todo o país. Toda a planta exala odor semelhante ao do limão, que se torna mais

Recebido para publicação em 22/10/2010

Aceito para publicação em 17/08/2011

Rev. Bras. PI. Med., Botucatu, v.14, n.2, p.352-357, 2012. 
intenso depois que a planta seca (Martins et al., 2000; Imig \& Zanco, 2008). Esta espécie é utilizada popularmente para controlar crises nervosas, taquicardia, melancolia, histerismo e ansiedade (Haber et al., 2005).

O óleo essencial está presente nos tricomas secretores das folhas e flores. Apresenta os compostos $\alpha$ e $\beta$ citral como majoritários, sendo estes os de maior interesse pelas indústrias farmacêuticas devido à atividade antioxidativa, antimicótica, antifúngica, sedativa e antivirótica (Teske \& Trentini, 1997; Haber et al., 2005; Lorenzi \& Matos, 2008).

Muitos estudos estão sendo realizados acerca da produção de compostos químicos vegetais, devido à alta demanda dos produtos sintéticos extraídos a partir desses produtos. Trabalhos realizados com cultivos sob malhas coloridas e sob proteção do excesso de irradiação solar tem sido desenvolvidos a fim de aumentar a produtividade do composto de interesse comercial (Brant et al., 2009).

A partir de trabalhos de otimização de cultivo, a melissa pode vir a ser uma espécie potencial em termos de produtividade de óleo essencial devido a abundância de tricomas secretores presentes na epiderme foliar (Martins \& Pastori, 2004).

Segundo Pinto et al., (2007), cada espécie responde de maneira distinta ao sombreamento. A variabilidade genética e o tipo de fotossíntese detém forte influência sobre tal produtividade (Taiz \& Zeiger, 2009). A adaptação das plantas às condições climáticas submetidas também influencia na quantidade do princípio ativo de interesse (Pinto et al., 2007).

Dentre esses fatores, a intensidade luminosa, a qualidade espectral, o fotoperíodo, a temperatura e o estresse hídrico podem influenciar no desenvolvimento vegetal, causando alterações morfofisiológicas. Podem ocorrer também modificações no aparato fotossintético de forma a promover acúmulo eficiente de massa seca, bem como, alterações no teor e na composição de metabólitos especiais (Alvarenga et al., 2003). O objetivo deste trabalho foi avaliar a variação no teor de óleo essencial, crescimento e produção de fitomassa de Melissa officinalis $\mathrm{L}$. influenciada pelo sombreamento.

\section{MATERIAL E MÉTODO}

O experimento foi realizado no Horto Medicinal do Instituto de Ciências Agrárias da Universidade Federal de Minas Gerais (ICA/UFMG), localizado no município de Montes Claros (645 m de altitude), norte de Minas Gerias (16 ${ }^{\circ} 40^{\prime} 50,92^{\prime \prime} \mathrm{S}$ e $43^{\circ} 50$ '22,36"W), no período de fevereiro de 2010 a junho de 2010. O clima é do tipo tropical semi-úmido quente, com temperatura média em torno de $25^{\circ} \mathrm{C} \mathrm{e}$ com estação seca prolongada.
O delineamento experimental utilizado foi o inteiramente casualizado, com quatro tratamentos e seis repetições. Os tratamentos consistiram em quatro níveis de radiação fotossinteticamente ativa (RFA), sendo: luz plena (testemunha) e sombreamento progressivo com tela tipo sombrite (sombreamento de 75,50 e $25 \%$ ). As mudas foram obtidas por estaquia e, após enraizamento, transplantadas para vasos com volume de $3,5 \mathrm{~L}$. O substrato utilizado foi coletado na camada superficial do solo $(0-20 \mathrm{~cm})$ (Souza et al., 2007) no Campus do ICA/UFMG. Os atributos químicos e físicos do solo determinados pela análise realizada pelo Laboratório de Solos do Instituto de Ciências Agrárias foram $\mathrm{pH}$ em água $=7,7 ; \mathrm{P}=35 \mathrm{mg}$ $\mathrm{dm}^{-3} ; \mathrm{K}=835 \mathrm{mmol}_{\mathrm{c}} \mathrm{dm}^{-3} ; \mathrm{Ca}=9,90 \mathrm{mmol}_{\mathrm{c}} \mathrm{dm}^{-3} ; \mathrm{Mg}=$ $3,70 \mathrm{mmol} \mathrm{dm}^{-3} ; \mathrm{Al}=0 \mathrm{mmol} \mathrm{dm^{-3 }} ;$ matéria orgânica= $12,90 \mathrm{~g} \mathrm{~kg}^{-1}$; areia grossa $=27,90 \mathrm{~g} \mathrm{~kg}^{-1}$; areia fina $=$ $38,10 \mathrm{~g} \mathrm{~kg}^{-1}$; silte $=24 \mathrm{~g} \mathrm{~kg}^{-1}$; argila $=10 \mathrm{~g} \mathrm{~kg}^{-1}$.

O solo foi passado em peneira de $4 \mathrm{~mm}$ e em seguida misturado com esterco bovino curtido também peneirado, na proporção $2: 1$, totalizando 350 $\mathrm{g}$ de esterco para cada $1050 \mathrm{~g}$ de solo por vaso. Posteriormente, foram transplantadas três mudas por vaso. Os vasos foram mantidos com umidade próxima à capacidade de campo. A temperatura foi estimada tomando-a a partir de cinco folhas expostas ao sol em cada parcela. As folhas para esta finalidade foram escolhidas aleatoriamente, sendo considerada a temperatura média entre elas.

$\mathrm{Na}$ colheita aos 80 dias após o transplantio foram determinados: o diâmetro do caule $(\mathrm{cm})$, a altura da planta $(\mathrm{cm})$, massa fresca e seca da parte aérea (g), o teor de óleo essencial (\%) em relação à base seca (massa), produção de óleo mg (massa total de óleo por planta fresca) e área foliar $(\mathrm{cm})$ (nas cinco folhas mais expandidas das plantas).

A extração do óleo foi realizada no Laboratório de Plantas Medicinais do ICA/UFMG, onde as folhas frescas foram hidrodestiladas por 3 horas em aparelho do tipo Clevenger, adaptado a balão de fundo redondo com capacidade de $1.000 \mathrm{~mL}$ (Ming et al., 1996). Para obtenção da massa seca, o material foi colocado em estufa de circulação forçada de ar, à temperatura de $60^{\circ} \mathrm{C}$, onde permaneceu até atingir peso constante. Os dados foram submetidos à análise estatística de variância e posteriormente à análise de regressão a 1 e $5 \%$ de probabilidade de erro para comparação da influencia do sombreamento no crescimento de melissa (Ribeiro Júnior, 2001).

\section{RESULTADO E DISCUSSÃO}

A tela sombrite ${ }^{\circledR}$ é disponível no comércio com indicação, pelo fabricante, de diferentes percentuais de sombreamento conforme o tipo de sombrite; no entanto, tais valores não apresentam correspondência com a RFA. Cassol et al. (2007) e 
Brant et al. (2009) não observaram efeitos da intensidade luminosa em Melissa officinalis em relação à taxa fotossintética.

Brant et al. (2009) observaram que o sombreamento em $50 \%$ beneficiou a produção e desenvolvimento da planta. Para Martins et al. (2000) em práticas empíricas de cultivo, observaram que a espécie apresenta bom desenvolvimento em meia sombra. Almeida et al. (2005), em cultivo experimental de Jacaranda puberula, fizeram a mesma observação, onde o melhor desempenho e sobrevivência das mudas foram em ambientes sombreados, similar ao submetido a espécie em ambiente natural devido a ocorrência de clareira em estágios sucessionais. Pode-se inferir que o aquecimento uniforme entre os tratamentos está relacionado ao aumento do rendimento quântico, que, por sua vez, indica a eficiência fotossintética representando uma resposta à temperatura ótima para a planta que possui fortes componentes genéticos e fisiológicos (Figueirôa et al., 2004).

Dos resultados observados, as características diâmetro do caule e altura da planta não apresentaram diferenças significativas entre os tratamentos. Haber et al. (2005), em experimento com cultivo hidropônico de melissa, observaram melhor desempenho da altura no início do canal de cultivo em relação as plantas dispostas na porção terminal. Os autores inferiram a melhor absorção de nutrientes da solução nutritiva no início do canal. Meleiro \& Graziano (2007) apresentaram resultados similares, não observando interferência significativa na espessura do diâmetro para o cultivo de tapeinóquilo (Tapeinochilos ananassae) em diferentes níveis de sombreamento, telado sombrite $50 \%$, telado duplo sombrite $50 \%$, estufa PEBD e a pleno sol.

Nas características massa fresca, massa seca e teor de óleo essencial, os tratamentos apresentaram similaridade na produção diferenciando apenas do tratamento a pleno sol, onde (MF): $25 \%=50 \%, 91,33 \mathrm{~g}$ e $68,66 \mathrm{~g}$; (MS): $25 \%=0 \%=50 \%$, $11,35,11,66,10,66 \mathrm{~g}$ e $4,33 \mathrm{~g}$ e TO: $25 \%=50 \%, 0,48$ e $0,40 \%$ respectivamente (Tabela 2 e Figuras 1, 2, e

TABELA 1. Médias da temperatura foliar do cultivo de Melissa em quatro níveis de sombreamento.

\begin{tabular}{ccc}
\hline Tratamentos & Sombreamento & Médias $^{*}$ \\
\hline 1 & $0 \%$ & $22,34 \mathrm{bc}$ \\
2 & $25 \%$ & $21,81 \mathrm{c}$ \\
3 & $50 \%$ & $23,45 \mathrm{a}$ \\
4 & $75 \%$ & $22,97 \mathrm{ab}$ \\
$\mathrm{CV}(\%)$ & & 1,953 \\
\hline
\end{tabular}

*Médias seguidas pela mesma letra não diferenciam estatisticamente pelo teste Tukey, a $5 \%$ de probabilidade.
3). Estes resultados evidenciam que a planta cultivada sob tela tipo sombrite ${ }^{\circledR}$ aumenta a produtividade à medida que diminui a densidade do fluxo radiante.

A área foliar em cultivo a $25 \%$ apresentou comportamento diferente dos demais tratamentos com $5,73 \mathrm{~cm}$. Resultado semelhante foi observado por Aguildera et al. (2004) em cultivo de botão-deouro (Siegesbeckia orientalis) sob duas condições luminosas (pleno sol e sombrite). Os autores observaram que as plantas apresentaram área foliar três vezes superior à planta mantida a plena luz. Este mecanismo desenvolvido pelas plantas é para alcançar rápido aumento da superfície fotossintetizante como forma de assegurar o aproveitamento das baixas intensidades luminosas (Pedroso \& Varela, 1995).

Em relação à massa seca, o sombreamento de $75 \%$ apresentou uma diferença muito grande em comparação aos demais tratamentos (Tabela 2 e Figura 2). Esta observação deve-se à fotoinibição e à capacidade fotossintética da melissa que pode ser severamente reduzida quando as plantas são expostas a altos níveis de radiação, superiores aos requeridos para saturar a fotossíntese (Pinto et al., 2007).

Silva \& Marenco (2001), em experimento com o capim-macho (Ischaemum rugosum), verificaram maior produção de massa seca ao menor nível de sombreamento (10\%). Dousseau e al. (2007) não observaram diferença estatística para massa seca no cultivo de Tapirira guianensis submetida aos níveis 0, 30, 50 e $70 \%$ de sombreamento. Pinto et al. (2007) encontraram resultados similares para alfazema-dobrasil (Aloysia gratissima) sob duas condições de cultivo (pleno sol e $40 \%$ de sombreamento). Gonçalves (2001) encontrou produção positiva para elixir-paregórico (Ocimum selloi) em pleno sol. Assim como Lima et al. (2008) para Caesalpinia ferrea 90 dias após o cultivo. Em guaco (Mikania glomerata) Castro et al. (2005) observaram maior produção de massa seca em cultivos sob sombreamento de $50 \%$. Para esses autores a resposta sobre o crescimento das plantas demonstra que as espécies respondem de maneira distinta ao sombreamento. $\mathrm{O}$ aumento da massa seca é devido à variação das divisões celulares e o padrão de expansão celular. Contudo, a deficiência de radiação no cultivo a $75 \%$ de sombreamento proporciona alongamento celular e estiolamento, sem alterar as massas fresca e seca (Alvarenga et al., 2003; Pinto et al., 2007).

O rendimento de óleo essencial apresentado pelos tratamentos 25 e $50 \%$ de sombreamento $(0,48$ e 0,40\%) foram superior ao esperado (Tabela 2 e Figura 3 ), pois de acordo com Sarer \& Kôkdil (1991) os percentuais variam entre 0,06 a $0,375 \%$. Entretanto a produção de óleo é proporcionalmente relacionada à capacidade metabólica da planta submetida à condições ideais de sombreamento. 
TABELA 2. Valores médios das características (AF) área foliar, (AL) altura da planta, (DC) diâmetro do caule, (MF) massa fresca, (MS) massa seca, (TO) teor de óleo essencial com base na massa seca em melissa (Melissa officinalis) cultivada em quatro níveis de sombreamento proporcionado pela tela tipo sombrite em Montes Claros - MG

\begin{tabular}{ccccccc}
\hline $\begin{array}{c}\text { Sombreamento pela } \\
\text { tela tipo sombrite (\%) }\end{array}$ & $\mathbf{A F}(\mathbf{c m})$ & $\mathbf{A L}(\mathbf{c m})$ & $\mathbf{D C}(\mathbf{m m})$ & $\mathbf{M F}(\mathbf{g})$ & $\mathbf{M S}(\mathbf{g})$ & TO (\%) \\
\hline 0 & $2,58 \mathrm{~b}$ & $24,1 \mathrm{a}$ & $0,43 \mathrm{a}$ & $57,5 \mathrm{bc}$ & $11,66 \mathrm{a}$ & $0,20 \mathrm{~b}$ \\
25 & $5,73 \mathrm{a}$ & $28,0 \mathrm{a}$ & $0,68 \mathrm{a}$ & $91,33 \mathrm{a}$ & $11,35 \mathrm{a}$ & $0,48 \mathrm{a}$ \\
50 & $2,26 \mathrm{~b}$ & $21,7 \mathrm{a}$ & $0,49 \mathrm{a}$ & $68,66 \mathrm{ab}$ & $10,66 \mathrm{a}$ & $0,40 \mathrm{ab}$ \\
75 & $3,21 \mathrm{~b}$ & $23,3 \mathrm{a}$ & $0,69 \mathrm{a}$ & $28,66 \mathrm{c}$ & $4,33 \mathrm{~b}$ & $0,00 \mathrm{c}$ \\
\hline $\mathrm{CV}$ & 27,7 & 29,62 & 29,9 & 29,06 & 35,17 & 53,7 \\
\hline
\end{tabular}

Médias seguidas pelo menos uma mesma letra minúscula, na coluna, não diferem significativamente pelo teste de Tukey, a $5 \%$ de probabilidade.

Gomes et al. (2009), ao trabalharem com Lippia citriodora, observaram que os tratamentos em pleno sol e com $30 \%$ de sombreamento apresentaram maiores teores de óleo essencial, $0,16 \%$ e $0,11 \%$, respectivamente. Infere-se a este resultado que o aumento no teor de óleo é devido ao elevado número de tricomas glandulares na epiderme, na qual é produzido pela ativação das rotas do metabolismo secundário da planta quando exposta a alta intensidade luminosa (Pinto et al., 2007).

Li et al. (1996), ao trabalharem com Thymus vulgaris, observaram que as plantas cultivadas sob luz plena apresentaram maiores teores de óleo essencial. Resultados similares foram encontrados para as espécies Lippia alba (Ventrella \& Ming, 2000) e Bacharis trimera (Silva, 2006). O presente resultado evidencia que o desenvolvimento e a produção de óleo essencial de muitas espécies aromáticas como citadas pelos autores são influenciados por fatores intrínsecos e extrínsecos como luz, água, temperatura foliar, quantidade de fótons irradiados e condições edáficas, influindo nos níveis fotossintéticos de cada espécie (Pedroso \& Varela, 1995; Gobbo-Neto \& Lopes, 2007; Morais et al., 2007).

Os tratamentos a $25 \%$ e $50 \%$ de sombreamento apresentaram a melhor produção de fitomassas fresca e seca e teor de óleo essencial (Tabela 2 e Figuras 1, 2 e 3). Pinto et al. (2007) não observaram diferença significativa entre os tratamentos pleno sol e $40 \%$ de sombreamento em cultivos de alfazema-do-brasil (Aloysia gratissima Gillies \& Hook Tronc.). Gonçalves et al. (2003) encontraram resultados similares para as plantas de elixir-paregórico (O. selloi), não divergindo estatisticamente, a pleno sol e sombreamento de $50 \%$.

Entretanto, o tratamento com $75 \%$ de sombreamento apresentou menor desenvolvimento para as características massa seca, fresca e teor de

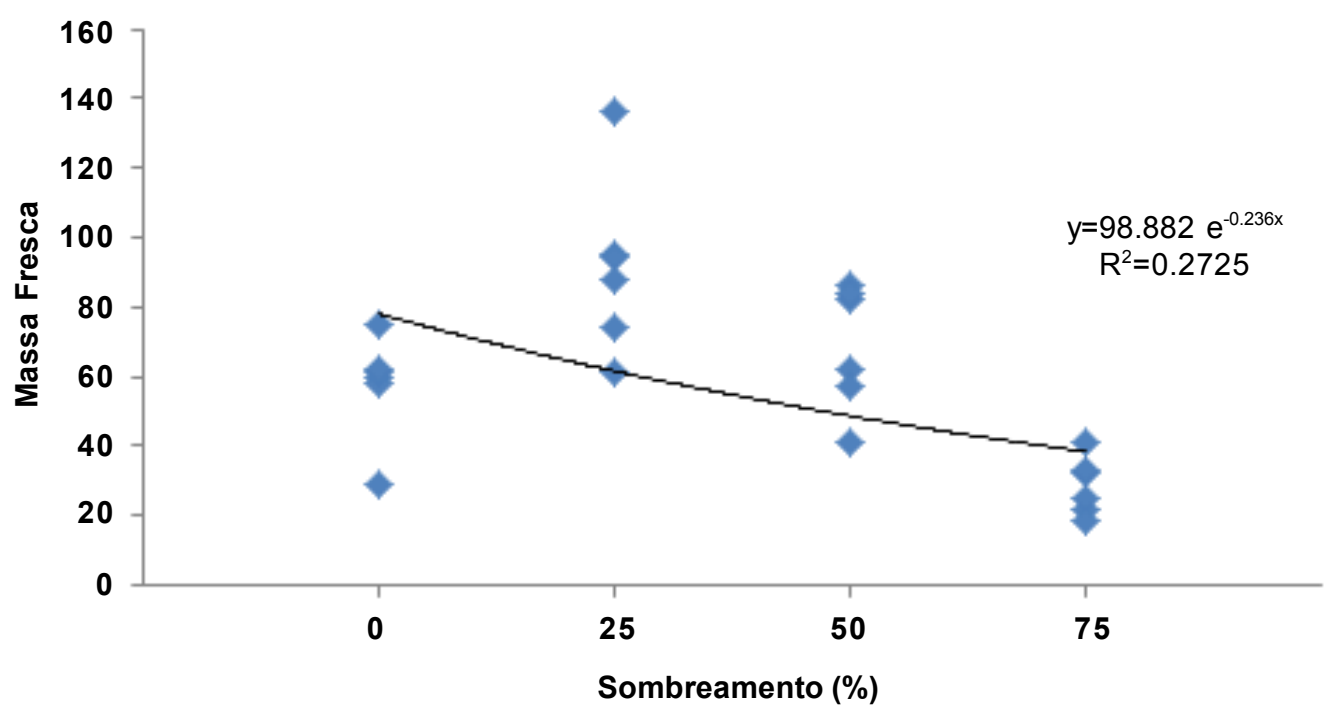

FIGURA 1. Regressão exponencial da massa fresca de melissa em função do sombreamento a $1 \%$ de propabilidade de erro $p=(0,0088)$. 


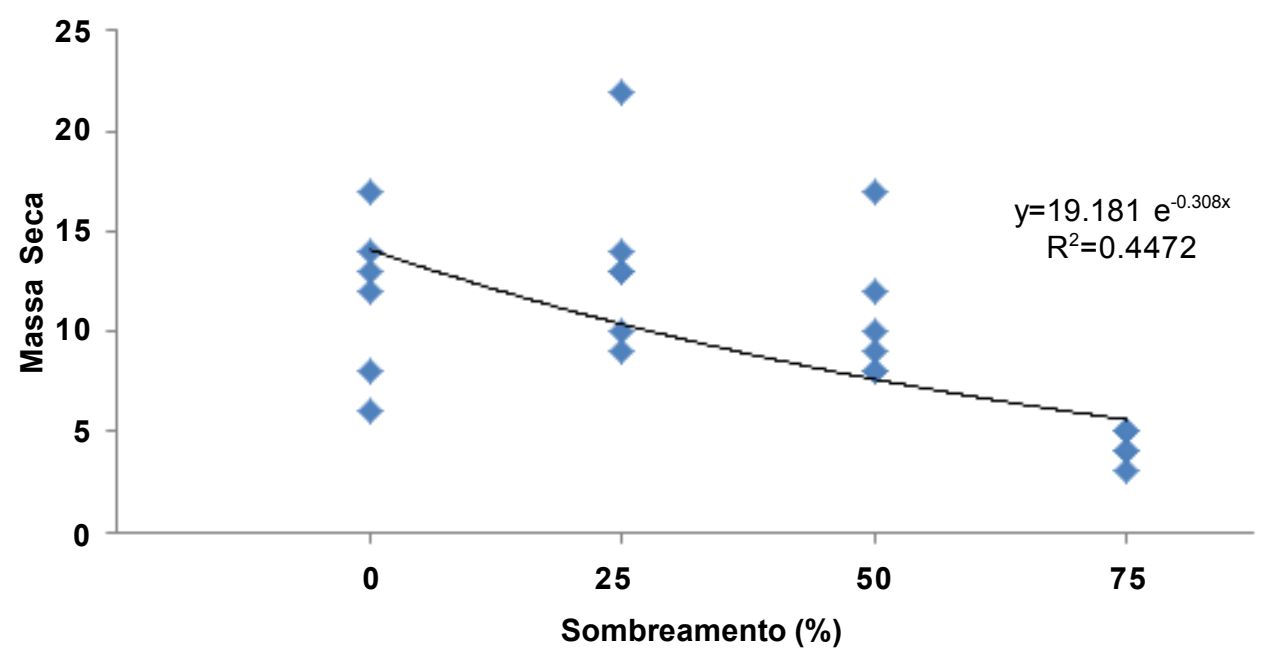

FIGURA 2. Regressão exponencial da massa seca de melissa em função do sombreamento a $1 \%$ de propabilidade de erro $p=(0,0003)$.

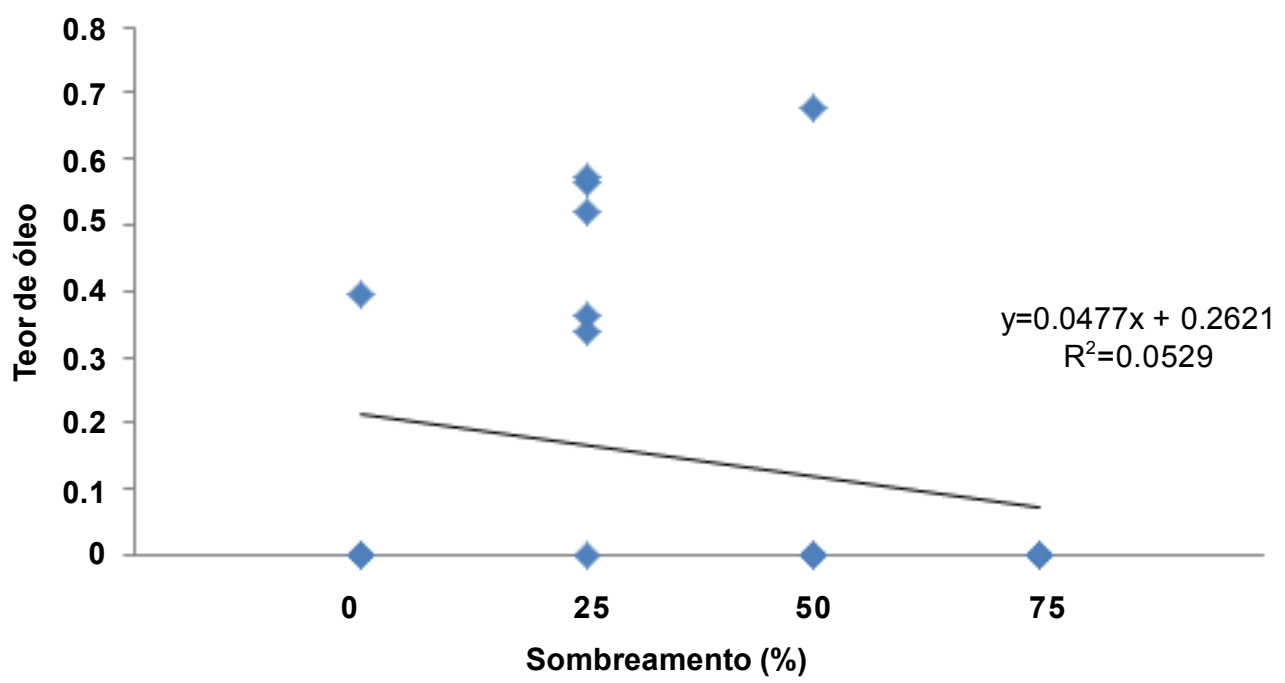

FIGURA 3. Regressão linear simples do teor de óleo essencial em função do sombreamento a $5 \%$ de probabilidade de erro $p=(0,2797)$.

óleo essencial (Tabela 2). Resultado oposto foi observado por Alvarenga et al. (2003) em cultivo de cróton (Croton urucurana). Segundo os autores, a maior produção de massa seca foi observada no tratamento com $30 \%$ de luminosidade.

Os resultados observados nesse trabalho mostram que plantas de melissa têm a produção de massa influenciada pela radiação. O tratamento a $75 \%$ de sombreamento não apresentou resultado satisfatório em todas as variáveis observadas. Ocorreu diferença estatística apenas para a massa seca sendo a menor produção do experimento com $4,33 \mathrm{~g}$. O tratamento a $25 \%$ de sombreamento apresentou as maiores médias de produção de massas, área foliar e teor de óleo essencial. Os demais tratamentos não apresentaram diferença estatística entre si. Infere-se que a redução de luminosidade no tratamento de $75 \%$ de sombreamento ficou aquém do ponto de saturação luminosa, reduzindo o processo fotossintético e a produção de massa.

\section{CONCLUSÃO}

Nas condições norte-mineiras de Fevereiro a Junho, o cultivo da Melissa officinalis pode ser conduzido em condições de até $50 \%$ de sombreamento, favorecendo a produção de massa seca, fresca e teor de óleo essencial, sendo estas variáveis de interesse comercial pelos produtores de plantas medicinais e aromáticas. Não existiu correlação linear ou exponencial entre os níveis de radiação testados e os parâmetros de produtividade avaliados. 


\section{REFERÊNCIA}

ALMEIDA, L.S. et al. Crescimento de mudas de Jacaranda puberula Cham. em viveiro submetidos a diferentes níveis de luminosidade, Ciência Florestal, v.15, n.3, p.323-9, 2005.

ALVARENGA, A.A. et al. Effects of different light levels on the initial growth and photosynthesis of Croton urucurana Baill. In: southeastern Brazil, Revista Árvore, v.27, n.1, p.53-7, 2003.

AGUILDERA, D.B. et al. Crescimento de Siegesbeckia orientalis sob diferentes condições de luminosidade.

Plantas Daninhas, v.22, n.1, p.43-51, 2004. Disponível em: <http://www.scielo.br/pdf/pd/v22n1/20144.pdf>. Acesso em: 15 Nov. 2010.

BRANT, R.S. et al. Crescimento, teor e composição do óleo essencial de melissa cultivada sob malhas fotoconversoras. Ciência Rural, v.39, n.5, p.1401-7, 2009. CASSOL, D. et al. Fotossíntese em Mentha piperita e Melissa officinalis sob sombreamento. Revista Brasileira de Biociências, v.5, n.2, p.576-8, 2007.

CASTRO, E.M. et al. Aspectos anatômicos e fisiológicos de plantas de guaco (Mikania glomerata Sprengel) submetidas a diferentes fotoperíodos. Horticultura Brasileira, v.23, p.846-50, 2005.

FIGUEIRÔA, J.M. et al. Crescimento de plantas jovens de Myracrodruon urundeuva Allemão (Anacardiaceae) sob diferentes referentes regimes hídricos. Acta Botânica Brasílica, v.18, n.3, p.573-80, 2004.

DOUSSEAU, S. et al. Influência de diferentes condições de sombreamento sobre o crescimento de Tapirira guianensis Alb. Revista Brasileira de Biociências, v.5, n.2, p.477-79, 2007.

GOBBO-NETO, L.; LOPES, N.P. Plantas Medicinais: fatores de influência no conteúdo de metabólitos secundários. Química Nova, v.30, n.2, p.374-81, 2007. GOMES, P.A. et al. Influencia do sombreamento na produção de biomassa, óleo essencial e quantidade de tricomas glandulares em cidrão (Lippia citriodora Lam.). Biotemas, v.22, n.4, p.9-14, 2009.

GONÇALVES, L.A. et al. Produção e composição do óleo essencial de Alfavaquinha (Ocimum selloi Benth.) em resposta a dois níveis de radiação solar. Revista Brasileira de Plantas Medicinais, v.6, n.1, p.8-14, 2003. GONÇALVES, L.A. Ontogenia dos tricomas glandulares e influência da radiação solar no desenvolvimento e no teor de óleo essencial de Ocimum selloi Benth (Lamiaceae). 2001. 95p. Dissertação (Mestrado - Área de concentração em Botânica) - Departamento de Botânica, Universidade Federal de Viçosa, Viçosa.

HABER, L.L. et al. Diferentes concentrações de solução nutritiva para o cultivo de Mentha piperita e Melissa officinalis. Horticultura Brasileira, v.23, n.4, p.1006-9, 2005.

IMIG, C.D.; ZANCO, J.J. Teste de adubação para plantas com atividade medicinal. In: Relatório de projeto de pesquisa. Método de pesquisa em fertilidade do solo, protocolo: 2351, 2008, p.23. UNISUL, Tubarão - Santa Catarina.

LI, Y.L. et al. Effect of light level on essential oil production of sage (Salvia officinalis) and thyme (Thymus vulgaris) Acta Horticulturae, v.426, p.419-27, 1996.

LIMA, J.D. et al. Efeitos da luminosidade no crescimento de mudas de Caesalpinia férrea Mart. Ex Tul. (Leguminosae, Caesalpinoideae). Acta Amazônica, v.38, n.1, p.5-10, 2008.

LORENZI, H.; MATOS, F.J.A. Plantas medicinais do Brasil: nativas e exóticas. Nova Odessa: Plantarum, 2008. 544p.

MARTINS, E.R. et al. Plantas medicinais. 3.ed. Viçosa: UFV, 2000. p.136-7.

MARTINS, M.B.G.; PASTORI, A.P. Anatomia foliar com ênfase nos tricomas secretores e análise cromatográfica de óleo essencial de Melissa officinalis L. (Lamiaceae). Revista Brasileira de Plantas Medicinais, v.6, n.2, p.7782, 2004

MELEIRO, M.; GRAZIANO, T.T. Desenvolvimento de tapeinóquilo em diferentes condições de luminosidde. Revista Brasileira de Horticultura Ornamental, v.13, n.1, p. 63-72, 2007.

MING, L.C. et al. Yeld of essential oil and citral content in different parts of lemongrass leaves (Cymbopongon citratus (DC.) Stapf.) Poaceae. Acta Horticulturae, v. 426 p.555-9, 1996.

MORAIS, H. et al. Caracterização microclimática de cafeeiros cultivados sob malha de sombreamento e a pleno sol. Revista Brasileira de Agrometeorologia, v.15, n.2, p.133-42, 2007.

PEDROSO, S.G.; VARELA, V.P.. Efeito do sombreamento no crescimento de mudas de sumaúma (Ceiba pentandra (L.) Gaertn). Revista Brasileira de Sementes, v.17, n.1, p.47-51, 1995.

PINTO, J.E.B.P. et al. Espectros morfofisiológicos e conteúdo de óleo essencial de plantas de alfazema-doBrasil em função de níveis de sombreamento. Horticultura Brasileira, v.25, n.2, p.210-4, 2007.

RIBEIRO JÚNIOR, J.I. Análises estatísticas no SAEG. Viçosa: UFV, 2001. 301p.

SARER, E.; KÔKDIL, G. Constituints of the essential oil from Melissa officinalis L. Planta Medica, v.57, p.89-90, 1991.

SILVA, F.G. et al. Influence of radiation level on plant growth, yield, and quality of essential oil in carqueja. Ciência e Agrotecnologia, v.30, p.52-7, 2006.

SILVA, M.R.M.; MARENCO, R.A. Teor de nitrogênio em Ischaemum rugosum sob três níveis de sombreamento. Planta Daninha, v.19, n.1, p.33-7, 2001. ISSN 0100-8358. SOUZA, M.F. et al. Influência do sombreamento na produção de fitomassa e óleo essencial em alecrimpimentoa (Lippia sidoides Cham.). Revista Brasileira de Biociências, v.5, n.2, p.108-10, 2007.

TAIZ, L.; ZEIGER, E. Fisiologia vegetal. 4 .ed. Porto Alegre: ARTMED, 2009, 848p.

TESKE, M.; TRENTINI, A.M.M. Compêndio de fitoterapia. Paraná: Herbarium, 1997, 317p.

VENTRELLA, M.C.; MING, L.C. Produção de matéria seca e óleo essencial em folhas de erva-cidreira sob diferentes níveis de sombreamento e épocas de colheita. Horticultura Brasileira, v.18, p.972-4, 2000. 